\title{
Neural-tube defect risk assessment for individual pregnancies using alphafetoprotein and acetylcholinesterase test results
}

\author{
PW STRIKE, J SMITH
}

From the Royal Air Force Institute of Pathology and Tropical Medicine, Halton, Aylesbury, Bucks HP22 5PG

SUMMARY Pregnancies at risk of being associated with (open) neural tube defects can be identified by maternal serum and amniotic-fluid alphafetoprotein (AFP) concentrations in excess of established screening cut-off valves in conjunction with the results of an amniotic fluid electrophoretic acetylcholinesterase screening test. The risks associated with the AFP cut-off values are formulated in terms of a population screening programme. These "population risks" are of little value in the assessment of test results from individual "at-risk" pregnancies, especially when the observed AFP concentrations are in proximity to the "cut-off" values. A complete risk assessment procedure for individual pregnancies is described and presented in graphical form to permit immediate access by clinical staff.

The measurement of serum and amniotic-fluid alphafetoprotein (AFP) concentration along with an electrophoretic acetylcholinesterase screen of the amniotic fluid has become widely used to identify pregnancies at-risk of being associated with (open) neural-tube defects (NTD). ${ }^{2}$ The interpretation of such risks is complicated by a marked overlap between the AFP concentration observed in nonaffected pregnancies and those in NTD-affected pregnancies, as shown in Fig. 1. The overlap is more marked in maternal serum than in amniotic-fluid.

There is no clear transition from zero risk to absolute certainty of an affected pregnancy. Cut-off levels have been established for population screening purposes, ${ }^{12}$ these being designed to minimise the number of misclassifications - that is, they represent an optimal compromise between the number of healthy pregnancies subjected to unnecessary (and risk-associated) follow-up procedures, and those affected pregnancies that remain undetected in the screening programme. Mothers with serum AFP concentrations equal to, or greater than the established serum cut-off value represent a specific subpopulation for whom the risk of an NTDassociated fetus is sufficiently great to justify the risks inherent in performing an amniocentesis. Amniotic fluid AFP concentrations equal to or greater than the appropriate amniotic-fluid cut-off level define a "high-risk" subpopulation. The screening risks

Accepted for publication 7 June 1982

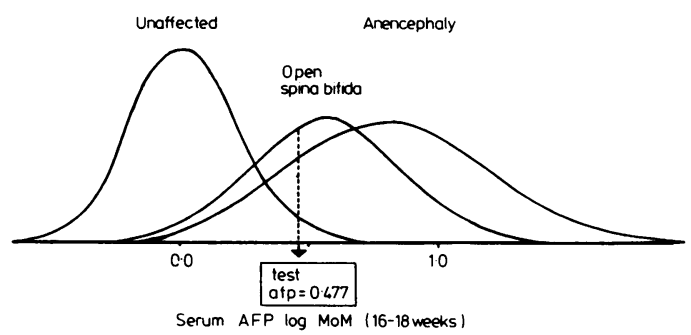

Fig. 1 Distribution of serum AFP values from unaffected, open spina bifida and anencephalic pregnancies at 16-18 wk of pregnancy. Note how the population picture is distorted by the absence of a scale on the vertical axis (probability density). Drawn to scale the NTD distributions would be vanishingly flat relative to the "unaffected" distribution, reflecting the prevalence of NTD pregnancies in the population.

associated with this high-risk subpopulation apply to all mothers with serum and amniotic fluid test values equal to or greater than their respective cut-off values-that is, they take no account of the exact AFP concentrations observed. The risks involved for an individual "high-risk" pregnancy are directly related to the actual AFP concentrations observed in both the serum and amniotic fluid - that is, AFP concentration in proximity to the screening cut-off values will be associated with substantially lower risk than AFP concentration well in excess of the cut-off values. 
Screening risk assessments are of value in planning an efficient screening service. They have no bearing upon the interpretation of risks for an individual pregnancy. This point has been made before. ${ }^{34}$ The procedure for evaluating individual risks, based on continuous test variables, is well established ${ }^{5}$ but the exact arithmetical steps involved are not widely known. The unfortunate consequences that will follow from a blanket application of screening probabilities (risks) to individual pregnancies associated with borderline rises in AFP (in serum and amniotic fluid), have prompted this communication.

\section{Material and methods}

The information required to perform an individual risk assessment falls into two categories: personal data and population data.

\section{PERSONAL DATA}

(a) The patient's estimated serum and amniotic fluid AFP values, along with the result of an acetylcholinesterase screening test are required. It is assumed that the amniotic fluid AFP result has been corrected for the effects of fetal blood contamination (should this be present). A provisional correction procedure has been described. ${ }^{2}$ The AFP measurements are subject to a marked interlaboratory variation, in recognition of which a reporting system based on multiples of locally established reference population medians (MoM) has been adopted. ${ }^{2}$

(b) An estimate of the gestational age is required, preferably supported by evidence from ultrasound scanning. Any history of previous NTD affected pregnancies in the subject mother should be noted.

\section{POPULATION DATA}

(a) The distributional characteristics of AFP in the population under study are required, for both serum and amniotic fluid, this data being conditional on gestational age. A log-normal approximation is employed for the AFP distributions in both serum and amniotic fluid, the adequacy of this approximation being established by Wald. ${ }^{6}$ Wald points out that the approximation is likely to hold over the range 1-5 MoM for serum AFP values and 2-4 MoM for amniotic fluid AFP values for the purpose of calculating individual risks. Exact distributional data has been supplied by Dr NJ Wald. ${ }^{6}$

(b) The prevalence of NTDs in the population under study is required. The UK prevalence is reported as 4-5 per 1000 births, ${ }^{3}$ with regional variations from 2 per 1000 (in the South-East), to 12 per 1000 (in Eire). Previous history of an NTD- affected pregnancy is associated with an increased prevalence (approximately tenfold with one affected child, twentyfold with two and fortyfold with three).

We assume that the imprecision of the AFP assay method used in the screening programme is similar to that used in establishing the population data of(a) above. Under this assumption, no account need be taken of the assay imprecision in the risk assessment procedure since this source of error is already incorporated in the distributional data itself.

(c) The proportion of NTD affected pregnancies giving positive acetylcholinesterase test results (true positives) and the proportion of non-NTD affected pregnancies giving positive acetylcholinesterase results (false-positives) are required for the subpopulation of mothers with (serum and) amniotic fluid AFP values in excess of the established "at-risk" cut-off values. The values used in this report have been abstracted from the report of the UK Collaborative Acetylcholinesterase study. ${ }^{7}$ A distinction is made between the proportions observed for fetally bloodstained amniotic fluid samples and those observed for maternally bloodstained or clear samples.

\section{CALCULATION OF INDIVIDUAL RISKS}

The risk assessment procedure is a straightforward application of Bayes' theorem for continuous variables ${ }^{8}$ the exact arithmetical steps involved being described in Appendix I. A computer implementation of the procedure (using a WANG 2200MVP system, Wang Laboratories Inc, Lowell, Mass, USA) has been used to generate a graphical substitute for the arithmetical calculations to permit immediate access to individual risk assessments. The graphs are presented in Figs. 2-4. The are specifically restricted to the assessment of singleton pregnancies with no previous history of NTD affected infants given an NTD prevalence of 4 per 1000 births in the population under study. Figure 2 provides a preliminary NTD risk given both serum and amniotic fluid AFP specimens drawn in the 16-18 wk gestation period. Figure 3 is appropriate when the serum AFP specimen is drawn at 16-18 wk gestation with the amniotic fluid AFP specimen drawn at 19-21 wk gestation. Any pregnancies not covered by these assumptions can be assessed independently using the full calculation procedures of Appendix I.

The use of these graphs is best described by example. Given a serum AFP concentration of 3.0 MoM (at 16-18 wk) and an amniotic fluid AFP of 3.6 MoM (also at 16-18 wk) we refer to Fig. 2 for the appropriate preliminary risk. The serum AFP value is located on the horizontal scale and the preliminary NTD risk read off the vertical scale from the intersection of the serum value with the appropriate 


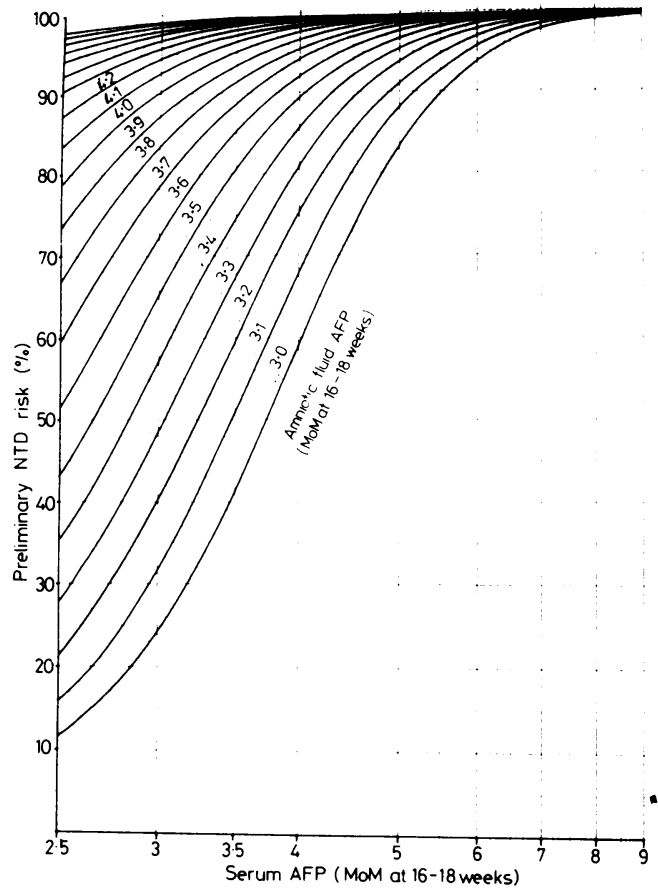

Fig. 2 Preliminary risk assessment graph for test subjects with no prior history of NTD affected pregnancies, given an NTD prevalence of 4 per 1000 births. Both serum and amniotic fluid AFP test specimens obtained at 16-18 wk gestation.

amniotic fluid AFP curve, the risk being approximately $72 \%$ for the above test data.

This individual risk contrasts markedly with the population screening risk of $\geqslant 97.5 \%$ associated with test AFP concentrations in excess of the screening cut-off values of $2.5 \mathrm{MoM}$ (for serum, 16-18 wk) and 3. $0 \mathrm{MoM}$ (for amniotic fluid, 16-18 wk).

The preliminary risk is now located on the horizontal scale of Fig. 4 (which is independent of the gestational dates) and the overall NTD risk located from the intersection of this value with the appropriate acetylcholinesterase test result curve. Continuing the example used above, a preliminary risk of $72 \%$ in conjunction with a positive acetylcholinesterase test results gives an overall risk of $99 \%$ if the amniotic fluid is clear (or stained with maternal blood only). The risk falls to $94 \%$ if the sample is stained with fetal blood.

In the presence of a negative acetylcholinesterase test result, the risk falls to around $1 \%$, irrespective of the sample bloodstaining status.

The graphs clearly reveal the interaction of the serum and amniotic fluid AFP concentrations in determining the risk for a given pregnancy, and the

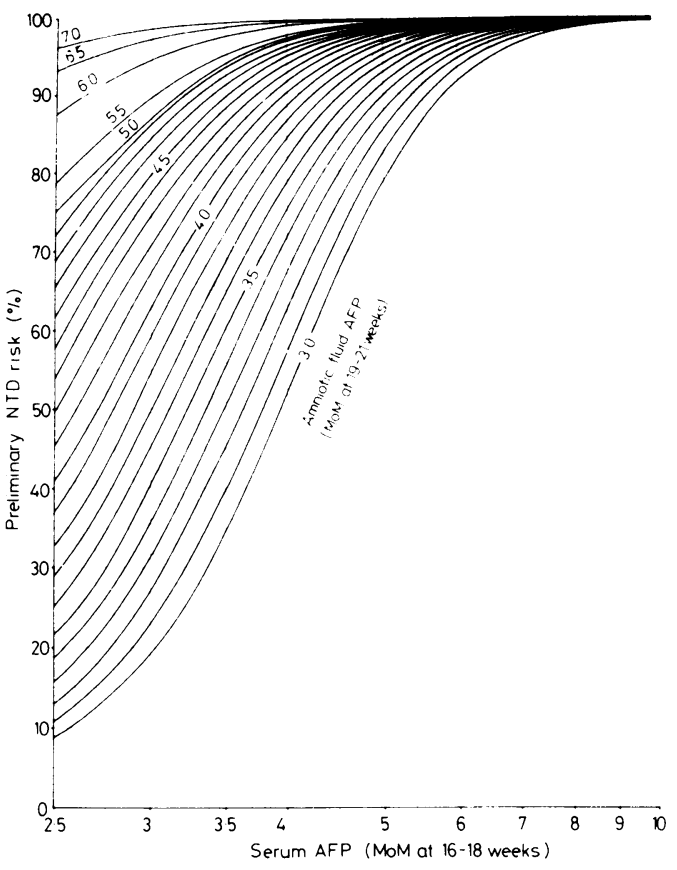

Fig. 3 Preliminary risk assessment graph for test subjects with no prior history of NTD affected pregnancies, given an $N T D$ prevalence of 4 per 1000 births. Serum AFP test specimen obtained at 16-18 wk gestation: amniotic fluid AFP test specimen obtained at 19-21 wk gestation.

discriminating power of the acetylcholinesterase test result in the interpretation of borderline AFP risk assessments. The loss of discrimination associated with fetal bloodstaining is also revealed graphically in Fig. 4.

The subpopulation of mothers with a previous history of NTD are associated with an increased prevalence if NTDs in subsequent pregnancies, this being taken fully into account in the calculation procedures of Appendix I. The graphical procedure presented in Figs. 2 and 3 are appropriate for the assessment of test subjects with no prior history of NTD affected infants.

Ultrasound scanning of the fetal spine and cerebral ventricles provides additional information on the status of individual pregnancies and is of particular value in the diagnosis of anencephaly. This has not been incorporated in the risk assessment procedure we describe, given the critical dependence of the information provided upon the skill of the sonar scan operator and the lack of population data on the utility of this procedure in the diagnosis of spina bifida. Given the availability of such data the overall NTD risk could be simply revised using the basic technique of stage III in Appendix I. 


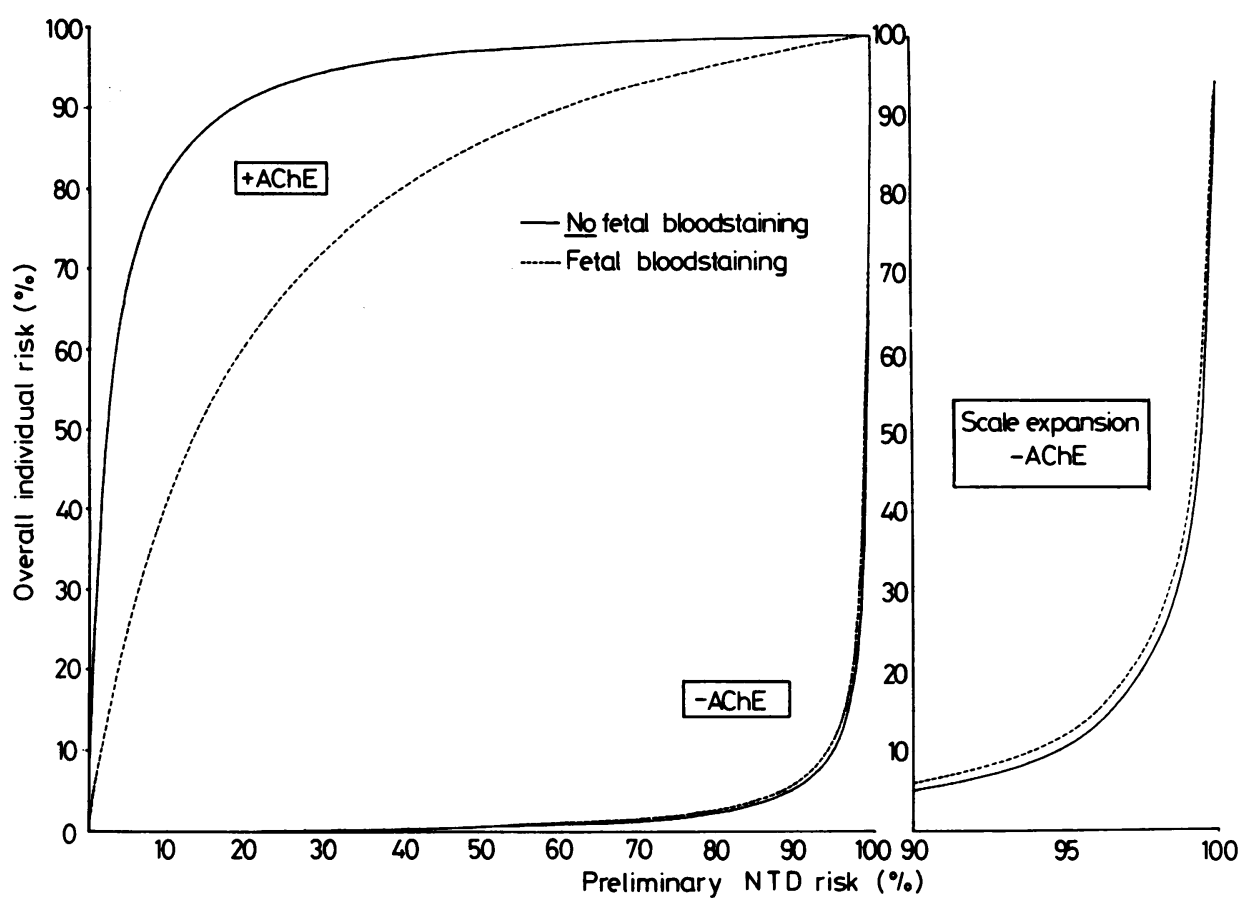

Fig. 4 Overall NTD risk assessment graph utilising the preliminary NTD risk in conjunction with the amniotic fluid AChE screening test result.

\section{Discussion}

The importance of assessing each patient individually in terms of their particular test results is brought out in Figs. 2 and 3. In terms of a population screening programme, all subjects with serum AFP concentrations in excess of $2.5 \mathrm{MoM}$ and amniotic fluid AFP concentrations in excess of $3 \mathrm{MoM}$ (at 16-18 wk gestation with no history of NTDs) are identified with risks in excess of $97.5 \%$ for an NTD affected pregnancy.

The risk for a particular test subject with AFP concentrations in excess of these cut-off levels is closely tied to the exact AFP concentrations recorded and Fig. 2 reveals a spectrum of risk ranging down to $11 \%$ for test values in proximity to the screening cut-off values. The acetylcholinesterase test result is highly effective in resolving the status of "equivocal risk" test subjects, although this can be compromised by the presence of fetal blood-staining in the amniotic fluid sample. Nonetheless, the risk assessment procedure described provides valuable support to the interpretation of individual test results as a basis for clinical action.

The use of probabilities in assessing individual cases demands a certain degree of common sense. In absolute terms the mother is either carrying an NTD affected fetus, or she is not. There is no uncertainty about that. The uncertainty lies in our perception of the true state of affairs obtaining for any given pregnancy. The procedures described are an efficient means of representing those uncertainties.

We thank Professor MJR Healy for his observations on the role of assay imprecision in the risk assessment procedure, Dr NJ Wald for his comments on the AFP risk assessment procedure and for supplying the distributional data for AFP in serum and amniotic fluid and the Director-General of Royal Air Force Medical Services for permission to publish this paper.

\section{Appendix I Calculation procedure}

\section{STAGE I}

To illustrate the calculation procedure we will adopt a serum AFP value of $3 \mathrm{MoM}$ at a gestational age of 16 wk, the pregnancy being singleton with no previous history of NTD affected infants. The prevalence of NTD is taken as being 4 per 1000 births for the purpose of this example.

Distributional data for serum and amniotic fluid AFP values for the gestational periods $16-18$ wk and 
19-21 wk are given by Wald. ${ }^{6}$

Distributional data for serum AFP in the conditions of interest at $16-18$ wk gestation are, in $\log _{10}$ MoM:

\begin{tabular}{lll}
\hline Condition & Mean & $S D$ \\
\hline Unaffected & $0 \cdot 0000$ & $0 \cdot 2163$ \\
Open spina bifida & $0 \cdot 5791$ & $0 \cdot 2697$ \\
Anencephaly & $0 \cdot 8143$ & $0 \cdot 3342$ \\
\hline
\end{tabular}

Let $\mathrm{x}_{\mathrm{i}}=\log _{10}$ (patient's serum AFP in MoM)

$$
=\log _{10} 3 \cdot 0=0 \cdot 477
$$

Calculate the standard normal deviate $\mathrm{Z}$ for each clinical state:

$$
\mathrm{Z}=\left(\mathrm{x}_{\mathrm{i}}-\text { mean }\right) / \mathrm{SD}
$$

We have: $Z_{\text {norm }}=(0.477-0.000) / 0 \cdot 2163=2 \cdot 205$

$$
\begin{array}{ll}
\mathrm{Z}_{\text {sp bif }} & =(0.477-0.579) / 0.2697=-0.379 \\
\mathrm{Z}_{\text {anen }} & =(0.477-0.814) / 0.3342=-1.009
\end{array}
$$

Calculate the normal density $f(Z)$ for each state using Appendix II. We will refer to these densities as "likelihoods."

$$
\begin{gathered}
\text { Likelihood } \\
\mathrm{f}\left(\mathrm{Z}_{\text {norm }}\right)=0.0351 \\
\mathrm{f}\left(\mathrm{Z}_{\text {sp bif }}\right)=0.3714 \\
\mathrm{f}\left(\mathrm{Z}_{\text {anen }}\right)=0.2397
\end{gathered}
$$

The next two steps are a straightforward application of Bayes' theorem. Multiply each likelihood by its associated prevalence, these being:

\begin{tabular}{lllll}
\hline Condition & $\begin{array}{l}\text { No history } \\
\text { of NTD }\end{array}$ & $\begin{array}{l}\text { One } \\
\text { previously } \\
\text { affected } \\
\text { infant }\end{array}$ & $\begin{array}{l}\text { Two } \\
\text { previously } \\
\text { affected } \\
\text { infants }\end{array}$ & $\begin{array}{l}\text { Three } \\
\text { previously } \\
\text { affected } \\
\text { infants }\end{array}$ \\
\hline $\begin{array}{l}\text { Unaffected } \\
\text { Open }\end{array}$ & 0.996 & 0.950 & 0.900 & 0.800 \\
spina bifida & 0.002 & 0.025 & 0.050 & 0.100 \\
Anencephaly & 0.002 & 0.025 & 0.050 & 0.100
\end{tabular}

For our example (no previous history) we have:

$\begin{array}{lrrrr} & \text { Likelihood } & \text { Prevalence } & \text { Product } \\ \text { Unaffected } & 0.0351 \times 0.996 & =0.03496 \\ \text { Open spina bifida } & 0.3714 \times 0.002 & =0.00074 \\ \text { Anencephaly } & 0.2397 \times 0.002 & =0.00048 \\ & & & \text { Sum }=.0 .03618\end{array}$

Calculate the individual risks (as probabilities) from the individual products divided by their sum:

$\mathrm{p}$ [Unaffected $\mid$ Serum AFP $=3 \mathrm{MoM}$ ] $=0.03496$ / $0.03618=0.966(96.6 \%)$

p [Spina bifida $\mid$ Serum AFP $=3 \mathrm{MoM}]=0.00074 /$ $0.03618=0.021(2 \cdot 1 \%)$

$\mathrm{p}[$ Anencephaly $\mid$ Serum AFP $=3 \mathrm{MoM}]=0.00046 /$

$0.03618=0.013(1.3 \%)$

With a serum AFP of $3 \mathrm{MoM}$, the exact risk of an NTD affected fetus is therefore $[2 \cdot 1+1 \cdot 3]=3 \cdot 4 \%$
To continue the example, we suppose an amniotic fluid AFP of 3.6 MoM to be obtained, this being in excess of the amniotic-fluid value cut-off value of $3 \mathrm{MoM}$ for 16-18 wk. Reassessment of the risks of an NTD affected fetus in this same mother follows in stage II.

The re-evaluation of the risks in stage II utilises the serum AFP "information" under the critical assumption that the serum and amniotic fluid AFP values are quite independent measures of risk. ${ }^{2}$

\section{STAGE II}

Reassessment of the risk of an NTD affected fetus in this same mother in light of the amniotic fluid AFP test result simply requires the repetition of the stage I calculations with the following substitutions.

(a) Distributional data for amniotic fluid AFP levels at $16-18 \mathrm{wk}$ are, in $\log _{10} \mathrm{MoM}$ :

\begin{tabular}{lll}
\hline Condition & Mean & $S D$ \\
\hline Unaffected & $0 \cdot 0000$ & $0 \cdot 1637$ \\
Open spina bifida & $0 \cdot 8633$ & $0 \cdot 2203$ \\
Anencephaly & $1 \cdot 3216$ & $0 \cdot 2145$ \\
\hline
\end{tabular}

Let $x_{i}=\log _{10}$ (Patient's amniotic fluid AFP in MoM) We assume the amniotic fluid AFP test result to be corrected for the effects of fetal blood contamination should this be present.

(b) The prevalence of the conditions given the patient's observed serum AFP values are taken directly as the individual risks (as probabilities) obtained in stage I.

To continue the example used in stage $I$, we assume an amniotic fluid AFP value of $3.6 \mathrm{MoM}$ (at $17 \mathrm{wk}$ gestation), which provides the following joint AFP risks (as probabilities);

$\mathrm{p}\left[\begin{array}{l|c}\text { Unaffected } & \begin{array}{c}\text { Serum AFP }=3 \mathrm{MoM} \\ \text { Am fluid AFP }=3 \cdot 6 \mathrm{MoM}\end{array}\end{array}\right]$ $=0.277(27 \cdot 7 \%)$

$\mathrm{p}\left[\begin{array}{l|c}\text { Spina bifida } & \begin{array}{c}\text { Serum AFP }=3 \mathrm{MoM} \\ \text { Am fluid AFP }=3 \cdot 6 \mathrm{MoM}\end{array}\end{array}\right]$ $=0.720(72 \cdot 0 \%)$

$\mathrm{p}\left[\begin{array}{l|c}\text { Anencephaly } & \begin{array}{c}\text { Serum AFP }=3 \mathrm{MoM} \\ \text { Am fluid AFP }=3.6 \mathrm{MoM}\end{array}\end{array}\right]$ $=0.003(0.3 \%)$

STAGE III

Reassessment of the NTD risks in light of the acetytcholinesterase test result requires the final two steps of the stage I calculations with the following substitutions:

(a) The prevalences are taken directly as the joint AFP risks (as probabilities) from the stage II calculations.

(b) The likelihoods are taken as the probabilities of observing a positive acetylcholinesterase test result in the three clinical states considered, conditional on the 
observation of amniotic fluid AFP values in excess of the screening cut-off value. These probabilities are taken from reference 7 .

$\left.\begin{array}{lll}\hline & \text { With fetal bloodstaining } & \text { No fetal bloodstaining } \\ \hline \text { Unaffected } & 0.162 & 0.025 \\ \text { Spina bifida } & 0.994 & 0.994 \\ \text { Anencephaly } & 0.996 & 0.996\end{array}\right\}$ likelihoods

Given a serum AFP of 3. 0 MoM and an amniotic fluid AFP of $3.6 \mathrm{MoM}$, the overall NTD risks given a positive $\mathrm{AChE}$ test result work out as follows:

$\begin{array}{lll} & \begin{array}{l}\text { fetal } \\ \text { blood- } \\ \text { staining }\end{array} & \begin{array}{l}\text { no fetal } \\ \text { blood- } \\ \text { staining }\end{array} \\ \mathrm{p} \text { [Unaffected }+\mathrm{AChE}]= & 0.059 & 0.010 \\ \mathrm{p} \text { [Spina bifida }+\mathrm{AChE}]= & 0.938 & 0.988 \\ \mathrm{p} \text { [Anencephaly } \mid+\mathrm{AChE}]= & 0.003 & 0.002\end{array}$

The risks associated with a negative acetylcholinesterase test result involve the same calculations using $(1-$ likelihood) in place of the likelihoods given above. For Fig. 4, the overall NTD risks are given as the sum of the spina bifida and anencephaly probabilities.

\section{Appendix II Standard normal densities}

We require the normal density $f\left(Z_{i}\right)$ for any given standard normal deviate $Z_{i}$. Tables of the standard normal integral (as opposed to density) tabulate areas under the curve-for example the area under the curve to the right of $\mathrm{Z}_{\mathrm{i}}$ in Fig. 1 . These would be appropriate to the computation of screening risk assessments where interest is focused on the risk associated with test values equal to or greater than a specified cut-off level $Z_{i}$.
Tabulations of the standard normal density function (the height of the curve at $\mathrm{Z}_{\mathrm{i}}$ ) are much less common and tend to be brief. A number of scientific calculators provide the function and the calculation procedure is easily implemented on a small programmable calculator. If you are in any doubt, use the example below as a test problem to check the values delivered by calculating devices.

$$
f\left(Z_{i}\right)=1 /\left[\sqrt{(2 \pi)} \times \exp \left(Z_{i}^{2} / 2\right)\right]
$$

For example, consider the normal density associated with $\mathrm{Z}_{\text {norm }}=2 \cdot 205$;

$$
\begin{aligned}
\mathrm{f}\left(\mathrm{Z}_{\text {norm }}\right) & =1 /\left[\sqrt{(2 \pi)} \times \exp \left(2 \cdot 205^{2} / 2\right)\right] \\
& =1 /[2 \cdot 5066 \times \exp (2 \cdot 43102)] \\
& =1 / 28.5010 \\
& =0.0351
\end{aligned}
$$

\section{References}

' Report of the UK collaborative study on alphafetoprotein in relation to neural tube defects. Lancet 1977; :1323-32.

${ }^{2}$ Second report of the UK collaborative study on alphafetoprotein in relation to neural tube defects. Lancet 1979;ii:651-62.

${ }^{3}$ Wald NJ, Cuckle HS. Alphafetoprotein in the antenatal diagnosis of open neural tube defects. Br J Hosp Med 1980;23:473-89.

4 Davidson RG, Walker WHC. Alphafetoprotein and antenatal screening. Clin Chim Acta 1980;105:1-3.

${ }^{5}$ Anderson JA, Boyle JA. Computer diagnosis: statistical aspects. Br Med Bull 1968;24:230-5.

- Fourth report of the UK collaborative study on alphafetoprotein in relation to neural tube defects. $J$ Epidemiol Commun Hith 1982;36:87-95.

${ }^{7}$ Report of the collaborative acetylcholinesterase study. Lancet 1981;ii:321-4.

Strike PW. Medical laboratory statistics. Bristol: J Wright \& Sons Ltd 1981:66-72.

Requests for reprints to: PW Strike, Royal Air Force Institute of Pathology and Tropical Medicine, Halton, Aylesbury, Bucks HP22 5PG, England. 